Stephan Bleek

\title{
Ein Wählermilieu in der Großstadt - Bemerkungen zum Durchbruch der SPD in einem Münchner Arbeiterviertel
}

Eine bislang nicht untersuchte Frage bei der Erforschung der Wählerbewegungen zur Sozialdemokratie während der Kaiserzeit betrifft die Herausbildung der Wāhlermilieus in den rasch wachsenden großstädtischen Arbeitervororten. Ich bin auf diese Frage in einer Fallstudie über das Münchner Westend eingegangen ${ }^{1}$, die Ergebnisse dieser Studie unterstreichen die Bedeutung sozial-moralischer Milieus ${ }^{2}$ in den Stadtquartieren für die Bildung und Stabilisierung der Wählerbasis der Partei.

München ist für die Wahlforschung ein außerordentlich wichtiges Untersuchungsfeld, stellt die Stadt doch einen ausgesprochenen Sonderfall für die Erfolgschancen der Sozialdemokratie im katholischen Umfeld dar, gewissermaßen einen roten Fleck auf der schwarzen Weste. Das Münchner Westend gehört zum Wahlkreis München II in dem Georg v. Vollmar schon 1884 erfolgreich war. Köln, ebenfalls eine Großstadt mit mehrheitlich katholischer Bevölkerung, befand sich zu diesem Zeitpunkt, auch was die Arbeiterwähler angeht, noch auf lange Sicht fest in der Hand des Zentrums ${ }^{3}$. Der Wahlkreis München II war nur zu etwa zwei Dritteln ein städtischer Kreis, er umfaßte auch große Teile des dörflichen Umlandes der Großstadt. Die städtischen Gebiete des Wahlkreises gehörten zu der Wachstumszone der Großstadt, wo sich vorwiegend seit den Gründerjahren rasch entwickelnde Arbeiterquartiere befanden. Die Bewohner dieser Quartiere waren großenteils Zuwanderer, die in der Masse aus Landgemeinden stammten und erst seit kurzem in der Stadt lebten und arbeiteten. Da es sich zu 90 Prozent um Angehörige der katholischen Konfession handelte, wāre zu vermuten, daß das Zentrum hier ähnlich günstige Bedingungen für den Gewinn von Arbeiterwählern vorgefunden haben sollte, wie es etwa im Rheinland oder auch in Oberschlesien der Fall war.

1 Stephan Bleek, Quartierbildung in der Urbanisierung. Das Münchner Westend 1890-1933 (Phil. Diss. München, MS 1986) im Druck.

${ }^{2}$ Zum Begriff M. Rainer Lepsius, Parteiensystem und Sozialstruktur: Zum Problem der Demokratisierung der deutschen Gesellschaft, in: Gerbard A. Ritter, Die deutschen Parteien vor 1918 (Köln 1973) 56-80.

${ }^{3}$ Hierzu Karl Robe, Katholiken, Protestanten und Sozialdemokraten im Ruhrgebiet vor 1914. Voraussetzungen und Grundlagen „konfessionellen“ und „klassenbewußten“ Wählens in einer Industrieregion, in: ders., Vom Revier zum Ruhrgebiet: Wahlen, Parteien, Politische Kultur (Essen 1986) 43-60, 44 . 
Die Wirkungen der Mobilität verdienen eine außerordentlich genaue Analyse, da komplexe Folgen für die Werthaltungen, Verhaltensweisen und eben auch die politischen Einstellungen der Beteiligten unterstellt werden können. Das Kernstück der Arbeit über das Münchner Westend bildete eine Analyse der Einwohnermeldebögen, die die räumlichen Bewegungen der Einwohner genau erfaßt haben. Die Bewohnerschaft des im wesentlichen zwischen 1875 und 1890 errichteten Stadtbezirks setzte zu dieser Zeit sich großenteils aus jungen Zuwanderern zusammen. Die Einwohnerzahl des Bezirks stieg von 7523 Personen im Jahr 1880 über 1885: 9841 auf 1890: 17405 Bewohner. Dann verlangsamte sich das Wachstum, um 1900 wurde mit 23000 Bewohnem der für lange Zeit gültige Höchststand erreicht. Eine bedeutende Gruppe unter den Einwohnem stellten die etwa 25 bis 40jährigen Zuwanderer, die nach einigen Jahren des Umherziehens eine Familie gegründet hatten und seßhafter geworden waren. Sie stellten vermutlich bis zu zwei Drittel der zum Reichstag Wahlberechtigten. Die Hochmobilen waren jünger, mehrheitlich unter 25 Jahren alt und ledig, sie pendelten oft noch saisonal zwischen der Stadt und dem Land oder aber wanderten über verschiedene Stādte weiter.

Das Westend war ein ausgesprochenes Arbeiterviertel. Die soziale Segregation der gesellschaftlichen Klassen war im München der Kaiserzeit ähnlich stark ausgeprägt wie in den meisten vergleichbaren Großstädten andernorts. Bedeutsam scheint dabei auch die Segregation zwischen zugewanderten und einheimischen Arbeiterschichten - die neuentstehenden Stadtbezirke nahmen die Masse der Zuwanderer auf, die Einheimischen, am Ort geborenen Unterschichtsangehörigen konzentrierten sich in den älteren Tagelöhner- und Handwerkervorstädten. Schließlich sei noch darauf hingewiesen, daß die Arbeiter dieser Epoche zumeist in enger räumlicher Verbindung zu ihren Fabriken wohnten, ein Stadtviertel wie das Westend, das in der Nähe des Bahnhofes und einigen Großbetrieben gelegen war, beherbergte also vor allem Eisenbahner und Arbeiter dieser Betriebe. Man kannte sich am Arbeitsplatz, traf sich aber auch nach Feierabend in der Nachbarschaft. Entscheidend war nun, daß die erwähnte, für die Analyse des Wählerpotentials der Parteien besonders interessante Einwohnergruppe sich als außerordentlich orts- und stadtviertelfest erwies: Durchschnittlich mehr als 25 Jahre verbrachten die Bewohner im selben Viertel. Umzüge wurden zumeist in allernächster Nachbarschaft vorgenommen. Diese Seßhaftigkeit band die Bewohner fest in ein bestimmtes Milieu ein, das sein eigenes soziales Profil entwickelte, das von einer starken altersmäßigen Homogenität geprägt wurde, das auf gleichgerichteten Erfahrungen vieler ruhte und das alle Voraussetzungen für besonders intensive Kommunikationsbeziehungen mitbrachte.

Wie erreichte die Sozialdemokratie nun hier ihre Erfolge? Die Zentrumspartei unternahm in München keineswegs geringere Anstrengungen, im Arbeitermilieu verankert zu bleiben, als etwa im Rheinland oder in anderen katholischen Regionen. Auch in München agierten „rote Kapläne“, wurde ein straff parochial organisiertes Vereinsnetz aufgezogen, wurde versucht, Bedingungen zu schaffen, die es zuließen, daß die neuen Erfahrungen in der großstädtischen Lebenswelt und der industriellen Arbeitswelt über am Glauben gebundene Deutungsmuster internalisiert wurden. Die politischen und weltanschaulichen Gegensätze zwischen Zentrum und SPD (oder auch 
zwischen Zentrum und Liberalen etc.) traten hier, im milden süddeutschen Klima, an der Wählerbasis keinesfalls mit geringerer Heftigkeit zutage als im rauhen Norden: die Wahlkämpfe wurden als zutiefst emotionsbeladene Schlachten ausgetragen, nicht selten kam es zu handgreiflichen Auseinandersetzungen. „Hie Christ! - hie Antichrist!“ - unter diesem Motto stand die Seelsorge in den beiden Pfarrgemeinden des Stadtbezirks. Bezeichnend für die intensiven seelsorgerischen Bemühungen ist vielleicht die Tatsache, daß im Westend die erste Kirche bereits einige Jahre vor dem ersten Schulgebauude geweiht wurde. Dennoch fand sich der politische Katholizismus bald in der Defensive. Das Zentrum konnte Anfang der 1880er Jahre noch bedeutende Teile der Münchner Arbeiterwähler an sich binden, allerdings war die „Partei der Nichtwähler" stark, besonders stark in den Zuwanderervierteln. Dies deutet darauf hin, daß trotz aller unternommenen Anstrengungen gerade hier besonders günstige Bedingungen für einen radikalen Bruch mit den hergebrachten politischen Wertesystemen und Normen vorhanden waren.

1884, als Georg v. Vollmar den Wahlkreis München II in der Stichwahl mit einem hauchdünnen Vorsprung von nur 115 Stimmen dem Zentrum abnehmen konnte, spielte gerade das Reservoir vormaliger Nichtwähler die ausschlaggebende Rolle. Beide Kandidaten konnten im jeweils für sie besonders günstig erscheinenden Umfeld in der Stichwahl kräftig zulegen. Während der Zentrumskandidat Westermayer in den Landgebieten des Wahlkreises seine Stimmenzahl mehr als verdoppeln konnte, in den städtischen Gebieten gerade einmal 8,7 Prozent mehr Wähler für sich zu mobilisieren vermochte, schaffte es Vollmar, in der Stadt um 116 Prozent zuzulegen und auf dem Land, wo er allerdings nur einen ganz geringen Anteil seiner Stimmen holte, sein Ergebnis ebenfalls deutlich zu steigem.

Die einfache Addition der Stimmen des in der Stichwahl nicht mehr beteiligten liberalen Kandidaten Fischer und derjenigen Vollmars scheint dessen Sieg auf den ersten Blick beinahe zu erklären. Bei näherer Betrachtung der Einzelresultate in den Wahllokalen zeigen sich jedoch komplexere Wählerwanderungen. Zwar erntete Vollmar mit Sicherheit liberale Proteststimmen gegen die Zentrumspolitik - so kommentierten auch die liberalen „Münchener Neuesten Nachrichten“: „Kolossales Anwachsen der Sozialdemokraten ... ist zunāchst ein Protest gegen die Ultramontanen ““ - der eigentlich entscheidende Faktor für ihn war jedoch die Mobilisierung vormaliger Nichtwāhler in den Arbeitervierteln wie dem Westend. Hier hatte der liberale Kandidat in der Hauptwahl nur magere 153 Stimmen ernten können, für Vollmar wurden 617 oder 47,2 Prozent der gültigen Stimmen abgegeben, für Westermayer 515 oder fast 40 Prozent. In der Stichwahl brachte es Vollmar auf 1009 Stimmen, wāhrend Westermayer leicht auf 511 Stimmen zurückfiel. Bei diesen Mobilisierungserfolgen zugunsten Vollmars zeigen sich bemerkenswerte Differenzierungen zwischen den Stadtvierteln. In der alten Unterklassenvorstadt Au oder im damals noch recht dörflichen Giesing wackelte der Zentrumsturm überhaupt nicht, Vollmar blieb unterdurchschnittlich erfolgreich. Der Einbruch in die Nichtwählergruppen gelang nur in den Zuwanderervierteln - den späteren Hochburgen der Sozialdemokratie. Am Rande sei

${ }^{4}$ Münchner Neueste Nachrichten, Jg. 37 (1884) Nr. 304 v. 29.10 .1884$. 
angemerkt, daß diese Weichenstellungen für 30, 40 und noch mehr Jahre von Bedeutung sein sollten: Die in den 1880er Jahren, in der Durchbruchsphase der SPD nicht eroberten Stadtviertel tendierten später in viel größerem Ausmaß zur KPD als die oft benachbarten Hochburgen der Sozialdemokratie. Solche Differenzierungen der Wählerkarte in der Stadt lassen sich sogar von Stimmbezirk zu Stimmbezirk, von Straße zu Straße verfolgen.

Bei Betrachtung der Resultate der folgenden Reichstagswahlen von 1887 und 90 fällt im Westend auf, daß die SPD nunmehr die Wählerschaft bereits in der Hauptwahl massenhaft für sich zu mobilisieren vermochte. 1887 stimmten hier 67 Prozent, 1890 sogar 79 Prozent der sich beteiligenden Wähler für Vollmar. Mit 1105 Stimmen erzielte er bereits in der Hauptwahl von 1887 ein besseres Ergebnis, als er in der Stichwahl von 1883 erreicht hatte - allerdings war das Elektorat in der Zwischenzeit weiter gewachsen $^{5}$. Überraschend ist nun, daß Vollmar in der Stichwahl - in der er den Wahlkreis an den Zentrumskandidaten Landes wieder verlor - keine weiteren Stimmen für sich zu mobilisieren vermochte. Die wenigen liberalen Stimmen kamen diesmal im wesentlichen wohl Landes zu gute, Vollmar gelang es nicht, Nichtwähler zu erreichen. Hier spielen sicherlich die besonderen Umstände der „Kartellwahlen“ eine Rolle, die Wahlbeteiligung war bereits in der Hauptwahl deutlich höher, als jemals zuvor. Für die spezifischen Bedingungen im Münchner Westend erscheint dennoch bemerkenswert, daß für die Sozialdemokratie nunmehr ein gewachsenes, aber auch weil die politischen Bedingungen dieser Wahl außerordentlich schwierig waren - stabileres, bereits im ersten Anlauf zu mobilisierendes Potential bereitstand. Dies wird man zum Teil, aber nicht ausschließlich mit dem Amtsbonus Vollmars erklären können. Die nunmehr viel entschiedenere, nicht mehr spontan zu nennende Stimmabgabe zugunsten der Sozialdemokratie deutet jedoch auch auf ein sich kräftigendes sozial-moralisches Milieu hin.

Der außerordentlich hohe Stimmenanteil von 1890 unterstreicht diese Interpretation. Vollmar erhielt nunmehr im Westend 2300 Stimmen, mehr als doppelt soviele wie 1887. Das Viertel war in den Jahren zuvor nochmals mit enormem Tempo gewachsen, das Elektorat hatte sich nahezu verdoppelt, darüberhinaus erreichte die SPD aber einen nochmals deutlich höheren Anteil an den im Viertel abgegebenen Stimmen - es waren fast 80 Prozent. Dieser erdrückende Übermacht deutet an, wieviele Möglichkeiten das Sozialistengesetz der Partei zuvor noch beschnitten hatte. Im Schatten der Halblegalitāt hatte sich das Wählermilieu jedoch längst formiert. Die losen parteipolitischen Orientierungen verfestigten sich nunmehr zum geschlossenen sozialistischen "Lager“. Massen von Zuwanderern wurden unmittelbar in die von der Sozialdemokratie geprăgte politische Kultur des Stadtviertels integriert, das Zentrum - es hockte nunmehr auf 420 Stimmen, weniger als 1883 erzielt wurden - war fast zu einer Randerscheinung degradiert, die allenfalls noch im engsten Kreis des katholischen Gesellenvereins oder in den Wohnungen der katholischen Baugenossenschaft „Rupertusheim“ Unterstützung fand. In den Wirtshäusern gaben sozialistisch orientierte Arbeiter den Ton an und übernahmen für die noch nicht mit der Stadt vertrau-

${ }^{s}$ Zu den Wahlberechtigten vgl. Bleek, Quartierbildung, Kap. 6, 5. 
ten Zuwanderer die Rolle der „Honoratioren“. Die Wählerorientierung im Westend bewegte sich jetzt für lange Zeit nicht mehr, die SPD blieb bis 1919, abgesehen von einem charakteristischen Einbruch 1907, die dominierende Partei des Viertels, deren Wāhlerschaft über die Arbeiterbevölkerung hinausreichte.

Ohne daß hier der Vorgang der Milieubildung im einzelnen nachgezeichnet werden kann, verdient festgehalten zu werden, daß die enorm rasche Integration so großer $\mathrm{Zu}$ wanderergruppen nur über die oben erwähnte strukturelle Homogenität der Bewohnerschaft zu erklären ist. Die spezifischen sozialräumlichen Bedingungen ermöglichten eine rasche sozialistische Orientierung mit Massenwirkung. Eine detaillierte Wahlkampfanalyse und die aus quellenbedingten Gründen leider kaum mehr mögliche Nachzeichnung der Agitationsformen und Mobilisierungswege würden die Vorgänge der Milieubildung erst detailliert erklären. Hier kam es darauf an, diesen bedeutsamen Vorgang ablesbar zu machen und damit auf mögliche weitere Forschungsarbeit hinzuweisen. 
